\title{
O TRABALHO EM EQUIPE NOS CENTROS DE ATENÇÃO PSICOSSOCIAL - CAPS
}

M aria A parecida G. Corrêa M ilhomem¹, A lice Guimarães B ottaro de Oliveira²

RESU M O: Objetiva-se analisar o trabal ho em equipe nos Centros deA tenção Psicossocial (CAPS), a partir da conceituação e tipologia de trabalho em equipe na área da saúde, buscando problematizar o tema no contexto da Reforma Psiquiátrica, à luz da categoria processo de trabalho do referencial teórico-metodológico da dialética marxista. Consideramos as condições deficientes de trabalho e suas conseqüências nas práticas das equipes que pretendem trabalhar na lógica da reabilitação psicossocial e concluímos que a compl exidade do trabal ho das equipes dos CA PS constitui um grande desafio que é acrescido das dificul dades impostas pela precarização do trabalho nos serviços públicos de saúde, especificamente nos serviços de saúde mental.

DESCRITORES: Saúde mental; Equipe de assistência ao paciente; Prestação de cuidados à saúde; Serviços comunitários de saúde mental; Trabalho.

\section{TEAM WORK AT PSY CHOSOCIAL CARE CENTERS - CAPS}

ABSTRACT : It aims to analyze teamw ork at Psychosocial Care Centers (CA PS, in Portuguese), founded on teamw ork conceptualization and typology in the health area, trying theme problematization under the context of the Psychiatric Reform, in the light of marxist dialetics on the work process category as the theoretical-methodological landmark. We consider that poor working conditions disrupt the daily routine of the team that intends to work in the field of psychosocial rehabilitation. We conclude that the work of the team in CAPS is complicated and creates a great challenge which is increased by the poor working conditions in the public health service, especially in mental health services.

DESCRIPTORS: M ental health; Patient care team; Health care delivery; Community mental health services; Work.

\section{EL TRABAJ O EN EQUIPO EN LOS CENTROS DE ATENCIÓN PSICOSOCIAL - CAPS}

RESU M EN: L a finalidad fue anal izar el trabajo en equipo en los Centros deA tención Psicosocial (CA PS), a partir de la conceptuación y tipología de trabajo en equipo en el área de salud, buscando enfatizar el tema en el contexto de la Reforma Psiquiátrica, a la luz de un proceso de trabajo dentro del referencial teórico - metodológico de la dialéctica marxista. Se consideraron las malas condiciones de trabajo y sus consecuencias en las prácticas de equi pos que pretenden trabajar en la lógica de la rehabilitación psicosocial. Se concluyó que la complejidad del trabajo en equipos CA PS constituye un gran desafío que se incrementa a las dificultades impuestas por la carencia de trabajo en los servicios públicos de salud, específicamente en los servicios de salud mental.

DESCR IPTORES: Salud M ental; Grupo de atención al paciente; Prestación de cuidados a la salud; Servicios comunitarios de salud mental; Trabajo.

\footnotetext{
${ }^{1}$ Enfermeira. Especialista em Saúde M ental. M estranda em Enfermagem pela Faculdade de Enfermagam. Universidade Federal do M ato Grosso FA EN/UFM T. Técnica da Secretaria M unicipal de Saúde de Cuiabá e Secretaria de Estado de Saúde de M ato G rosso.

${ }^{2}$ Enfermeira. Doutora em Enfermagem Psiquiátrica. Professora A ssociada e pesquisadora do Grupo A rgos e do Núcleo de Estudos em Saúde M ental da FAEN/UFMT.
} 


\section{INTRODUÇÃO}

A presentamos neste artigo uma reflexão sobre o trabalho em equipe nos Centros de A tenção Psicossocial (CAPS), a partir da conceituação e tipologia de trabalho em equipe na área da saúde e, especificamente, da saúde mental|(1-2), problematizando o tema no contexto da R eforma Psiquiátrica, à luz da categoria processo de trabalho, do referencial teóricometodológico da dialética marxista.

Situamos o trabalho e o trabal ho em saúde na perspectiva de sua construção histórico-social e, neste, o trabalho assistencial em saúde mental, numa contextualização histórica que parte do modelo médico-psiquiátrico ou asilar em direção ao modelo psicossocial que se pretende hoje, identificando os instrumentos de trabalho, com ênfase na equipe multidisciplinar. A nalisamos as premissas do trabalho dos CA PS a partir de suas definições normativo-legais e al gumas condições de trabal ho que são exigidas das equipes que pretendem trabalhar na lógica da reabilitação psicossocial.

Compreendemos que as práticas de saúde são articuladas ao modo de produção de uma dada sociedade, às políticas sociais, e que são componentes de um processo de trabalho coletivo, histórico e organizado social mente para atender aos carecimentos sociais ${ }^{(3)}$

\section{O TRABALHO EM SAÚDE}

O homem é um ser em permanente construção, que vai se fazendo no tempo pela mediação de sua prática, de sua ação. É, assim, um ser histórico, que vai se construindo no espaço social; não é uma realidade dada, mas fundamental mente um sujeito que vai construindo a sua realidade. Esta construção que o homem faz inicia-se com a sua relação com a natureza, que é a fonte da vida material e essa, por sua vez, cria novas necessidades ${ }^{(4)}$.

A o contrário dos demais seres vivos, esse intercâmbio homem/natureza não se dá de modo mecânico. As intervenções que os homens estabelecem com a natureza são marcadas por uma intencionalidade que antevê e projeta a sua ação. Esta ação humana sobre a natureza é o trabalho, compreendido como a prática produtiva essencial mente humana ${ }^{(4)}$.

Dadas as características do trabalho - produzir sempre face às necessidades sociais, relacionar os homens por meio de seus produtos e conforme o grau de domínio que têm (ou não têm) das condições de trabalho -, ele é, antes de tudo, o processo de produção e reprodução humanas ${ }^{(4)}$. Portanto, o trabalho humaniza o homem na medida em que proporciona mediações da sua existência com a natureza - 0 universo da vida material, mediações entre os homens e da relação consigo mesmo, que permitem a existência da sociedade, da cultura e da subjetividade, em suma, 0 universo social e cultural.

0 trabalho que atende às necessi dades de saúde é uma das mais antigas formas de trabal ho social. 0 trabalho em saúde, embora focalizado no corpo humano, nas suas dimensões objetivas e subjetivas, não pode ser abstraído de suas relações históricas. Não existe um processo de trabalho em saúde "em geral", porque a ele não corresponde um objeto "natural", já dado independente da história. A s necessidades de saúde, e conseqüentemente os objetos de trabal ho em saúde, são historicamente determinados ${ }^{(3,4)}$. A ssim, na assistência à saúde mental, ao longo de seus aproximadamente dois séculos de existência como campo técnico-científico, já se estruturaram diferentes objetos, instrumentos e finalidades e, atual mente, se organiza a assistência a partir de um determinado recorte de objeto - um sujeito que apresenta um sofrimento mental -, com a finalidade de aliviar o seu sofrimento e integrá-lo socialmente. Para isso, se organizam específicos instrumentos de trabalho, diferentes de outros anteriormente utilizados na história.

A o processo de trabalho em saúde corresponde: 1) um objeto/sujeito, que é a quem (ou para o que) se dirige o cuidado; 2) instrumentos ou meios que incluem conhecimentos, métodos, técnicas, equipamentos e/ou recursos para realizar esse trabalho; e 3) a finalidade ou o que se quer alcançar com o trabal ho. Essas três dimensões do trabalho em saúde não existem isoladamente, são interdependentes, se conformam mutuamente e, essa relação indissociável existe somente num dado contexto sócio-histórico, marcado pel os determinantes gerais do modo de produção capitalista que, por sua vez, condiciona a existência de uma sociedade de classes caracterizada pelo alto nível competitivo. É necessário ressaltar ainda que, no processo de trabalho em saúde, os agentes que operam o saber e as práticas são seres humanos, assim como os "objetos" aos quais as práticas/cuidados se dirigem. Essa característica incide diretamente na condição de execução de um 
determinado tipo de trabal ho: trabal ho vivo ou em ato ${ }^{(5)}$.

0 trabalho em saúde, em decorrência dessa característica de se realizar no encontro e por meio de atos entre sujeitos - trabal hador de saúde e usuário -, se dá pel a criação de espaços de rel ações e interação, que são momentos de produção e consumo de ações de saúde ${ }^{(5)}$.

\section{SAÚDE MENTAL: DO MODELO ASILAR AO MODELO PSICOSSOCIAL}

A assistência psiquiátrica vem sofrendo profundas mudanças nos países ocidentais desde a segunda guerra mundial. A princípio, buscou-se transformar internamente o asilo e o hospital psiquiátrico, na tentativa de humanizá-los e de torná-los terapêuticos e mais eficazes na recuperação das pessoas internadas, porém essas experiências logo revelaram suas limitações. A partir dos anos 60 surgiram propostas mais radicais, no movimento da anti-psiquiatria, que questionavam o saber psiquiátrico e seu mandato social em relação à loucura. A partir dos anos 70, principalmente na Itália, se consolidou outro movimento que até hoje vem se revelando desafiador, mas que apresenta experiências bem sucedidas e se amplia mundial mente: é a proposta da desinstitucional ização(2).

Coerente com as primeiras fases já referidas, a organização do trabalho para a assistência psiquiátrica se restringia, até 0 início do século $X X$, à prática médica baseada na classificação e descrição das "doenças mentais" e centralizava todo 0 arsenal de cura no hospício, expressão máxima da tecnologia médicopsiquiátrica (naquela época nomeada alienista). Essa organização, que também se denomina "modelo asilar"(6), contava com as práticas complementares de pessoal subalterno que visavam essencialmente 0 controle (vigilância) dos internados. Entre aqueles, identificavamse os primeiros cuidadores "enfermeiros" na assistência psiquiátrica ${ }^{(7)}$.

0 paradigma da desinstitucionalização, que influenciou significativamente o movimento de Reforma Psiquiátrica no Brasil, vai além da reforma das tecnologias assistenciais e redimensiona o conceito de "doença mental" no sentido epistemológico, sóciocultural e jurídico-legal. A ssim, nesse novo paradigma, enfatiza-se a reinvenção da saúde no sentido da produção devida, de uma sociabilidade que amplia e complexifica 0 objeto de conhecimento e intervenção (a pessoa que sofre mentalmente)(8).
Observa-se atualmente no Brasil e no mundo a reorientação do modelo assistencial que se pauta por uma concepção de saúdemental compreendida como processo e não como ausência de doença, na perspectiva de produção dequalidade de vida ${ }^{(9)}$ daquele que sofre. N esse modelo, os serviços devem ser territorializados e integrados à rede de saúde, real izando ações de proteção, promoção eassistência em saúde mental, além de incluir ações dirigidas aos familiares e comprometer-se com a construção de projetos de inserção social, respeitando as possibilidades individuais e os princípios de cidadania ${ }^{(10)}$.

\section{CAPS: uma nova tecnologia em saúde mental}

Buscando situar a origem desse dispositivo de cuidado na saúde mental que atualmente se apresenta como central na reorientação do modelo assistencial brasileiro, observa-se que o primeiro CAPS surgiu em 1987 - o CAPS Luis da Rocha Cerqueira ou CAPS I tapeva - naCidade deSão Paulo, no movimento político da redemocratização brasileira e da R eforma Sanitária. Esta criticava os conceitos, modalidades de atenção ede financiamento das ações de saúde (e de saúde mental), que se fortaleceu principalmente no final da década de 1980. N esse mesmo processo, os Núcleos de A tenção Psicossocial (NAPS) em Santos, também se constituíram como uma expressão significativa dessa nova tecnologia clínico-política na área da atenção à saúde mental. Os NAPS representaram o movimento mais efetivo de ruptura paradigmática com o modelo anterior hospitalocêntrico e excludente -, na medida em que se organizavam através do funcionamento territorial izado, diuturnamente e integrados à rede de serviços de saúde ${ }^{(10)}$.

Essas duas experiências subsidiaram a formulação, pelo M inistério da Saúde (M S), da Portaria n. 224 que, em 1992, estabelecia pela primeira vez critérios para o credenciamento e financiamento de CAPS no Sistema Ú nico de Saúde (SUS) (10).

Os CAPS são atualmente regulamentados pela Portaria n. 336/GM/2002 e integram a rede do SUS. Constituem-se como a principal estratégia utilizada no processo de R eforma Psiquiátrica, e são, por definição, "Iugar de referência e tratamento para pessoas quesofrem com transtornos mentais, [...] cuja severidade e/ou persistência justifiquem sua permanência num dispositivo de cuidado intensivo, comunitário, personalizado e promotor de vida"(11:13), e têm por objetivos: 
[...] oferecer atendimento à população de sua área de abrangência, realizando o acompanhamento clínico e a reinserção social dos usuários pelo acesso ao trabalho, lazer, exercício dos direitos civis e fortal ecimento dos laços familiares e comunitários. [...] criado para ser substitutivo às internações em hospitais psiquiátricos ${ }^{(11: 13)}$.

Portanto, pretende-se que os CA PS se consolidem como dispositivos eficazes na diminuição de internações em hospitais psiquiátricos e na mudança do modelo assistencial. Para isso, eles devem oferecer diversas atividades terapêuticas, como psicoterapia individual ou em grupo, oficinas terapêuticas, atividades comunitárias e artísticas, orientação e acompanhamento do uso de medicações, atendimento domiciliar e familiar, organizadas num projeto terapêutico individualizado, que tem por objetivo o tratamento e a reabilitação, estes entendidos como processos de ampliação da autonomia pessoal/social dos usuários.

Neste dispositivo estratégico de cuidado em saúde mental, as práticas terapêuticas devem romper com 0 modelo disciplinador tipicamente observado no modelo alienista/asilar e se constituir como uma trajetória dinâmica e flexível com o objetivo de enriquecer a existência do usuário/sujeito. Esteécompreendido como o protagonista principal de sua vida e tratamento, e assim - CAPS funciona como o principal agenciador de condições concretas para que este sujeito participe de trocas no âmbito social.

Observa-se, portanto, que, nesse dispositivo, a equipe multidisciplinar que tem por objetivo viabilizar esse tratamento/reabilitação, opera com conteúdos técnico-políticos que vão muito além das técnicas profissionais tradicionais do campo "psi". Para viabilizar esse projeto terapêutico ambicioso, énecessário romper com vários fundamentos do modelo médico-psiquiátrico: o conceito de doença mental como desrazão e erro, e a periculosidade que, por sua vez, determinam a necessidade do isolamento e de uma "pedagogia" para a reeducação rumo à uma suposta normalidade abstraída da pessoa ${ }^{(12)}$.

A s equipes operam, portanto, com elementos de uma "clínica ampliada", centrada no sujeito einseparável das formas de organização dos processos de trabalho e da política, pois os CAPS "colocam em especial evidência as inter-relações entre subjetividade, gestão dos processos de trabalho e clínica"(10:1056).

Visando refletir sobre esses desafios postos para as equipes dos CA PS, e considerando-os na historicidade do processo atual de organização das políticas de saúde no Brasil, apresentamos a seguir algumas definições sobre trabalho em equipe.

O trabalho na atenção psicossocial: enfocando a equipe

A maioria dos estudos sobre trabalho em equipe em saúde apresenta uma abordagem tecnicista, em que 0 trabal ho de cada profissional é enfocado como atribuições, tarefas ou atividades específicas e, assim, a equipe multiprofissional é apresentada como um conjunto de profissionais que operam diferentes práticas, sobre uma realidade dada, na qual as formas de articulação dos trabal hos específicos não é historicizada(1).

Tendo em vista a necessidade de problematizar essa conceituação detrabal ho em equipeno contexto da atenção psicossocial, apresentamos alguns conceitos e tipologias de trabal ho em equipe, a partir de dois estudos ${ }^{(1,2)}$.

Admitindo-se que o trabalho em saúde é coletivo, resultado da divisão histórica do trabal ho e que essa remete a uma determinada (e relativa) autonomia técnica "concebida como a esfera de liberdade de julgamento e de tomada de decisão frente às necessidades de saúde dos usuários"(1:107) que, por sua vez, se articula com uma determinada valoração social atribuída aos diferentes trabal hos/trabal hadores e que legitima essa autonomia técnica - divisão social e técnica do trabalho -, apresentamos a tipologia de trabalho em equipe proposta por Peduzzi ${ }^{(1: 106)}$ :

\begin{abstract}
E quipe agrupamento, em que ocorre a justaposição das ações e o agrupamento dos agentes, e equipe integração, em que ocorre a articulação das ações e a interação dos agentes. Em ambas, estão presentes as diferenças técnicas dos trabalhos especial izados e a desigualdade de valor atribuído a esses distintos trabalhos [...] e tensões entre as diversas concepções e os exercícios de autonomia técnica, bem como entre as concepções quanto a independências dos trabal hos especializados ou a sua complementaridade objetiva.
\end{abstract}

Portanto, ao definirmos como meta o trabal ho em equipe nos CA PS, não pode haver a pressuposição de uma idealizada homogeinização ou igualdade de saberes técnicos e valores sociais dos diferentes trabalhos profissionais, mas antes 0 enfrentamento dessas diferenças e desigualdades no processo cotidiano de trabalhar/cuidar. A equipe integração éa requerida no trabalho de atenção psicossocial para buscar a necessária articulação das ações, a interação rumo a uma concepção menos conflituosa de intencionalidades e metas, ou, em outros termos, a 
superação do isolamento dos saberes.

Essa construção necessita ser apreendi da como um processo dinâmico, assim, essa articulação requer um movimento consciente e ativo dos agentes para 0 reconhecimento das diferenças e desigualdades diferenças técnicas entre as profissões que são hierarquizadas e disciplinadas por meio de valores e normas sociais $s^{(1,13)}$, porém sem esquecer que tais diferenças são igualmente históricas, e em determinados momentos podem perder seu sentido, exigindo novas mudanças.

$M$ uitas vezes, as equipes multiprofissionais se constituem na modalidade agrupamento, como um conjunto de diferentes agentes, realizando processos de trabal ho parcelares e especializados (com objetos, saberes e instrumentos distintos), sob a autoridade do saber e da prática do médico ${ }^{(1,13,14)}$. E $m$ decorrência, se apresentam como arenas de conflitos numa justaposição de agentes e trabal hos alienados.

Num estudo que parte de uma concepção sistêmica e aborda a interdisciplinaridade no Serviço Social, Vasconcelos ${ }^{(2)}$ apresenta a seguinte tipologia:

Multidisciplinaridade ou multiprofissionalidade: conjunto de profissionais de diferentes áreas trabal hando isoladamente, embora num mesmo espaço institucional, operando simultaneamente diferentes saberes, sob uma coordenação apenas administrativa.

Pluridisciplinaridade: as práticas multiprofissionais são agregadas em al guns espaços e tempos de interlocução "científica", como discussões clínicas e estudos de caso com o objetivo de planejar e avaliar ações assistenciais.

Interdisciplinaridade auxiliar: "contribuições de uma ou mais disciplinas para o domínio de uma disciplina específica que se posiciona como campo coordenador das demais" (2:45). O bserva-se essas práticas em situações da psiquiatria contemporânea que, visando a manutenção da hegemonia médica no campo da atenção à saúde mental, "apropria-se de contribuições de outras disciplinas, de forma subordinada"(2:47).

Interdisciplinaridade:

É entendida como estrutural, havendo reciprocidade, enriquecimento mútuo, com uma tendência à horizontalização das relações de poder entre os campos. Exige a identificação de uma problemática comum, com levantamento de uma axiomática teórica e/ou política básica e de uma plataforma de trabalho conjunto, colocando-se em comum os princípios e os conceitos fundamentais, esforçando-se para uma decodificação recíproca da significação, das diferenças e convergências desses conceitos e, assim, gerando uma fecundação e aprendizagem mútua, que não se efetua por simples adição ou mistura, mas por uma recombinação de elementos ${ }^{(2: 47)}$.

Transdisciplinaridade: radicalização da interdisciplinaridade que tem como consequência a criação de um novo campo teórico ou disciplinar.

Observa-se que a interdisciplinaridade, tal como definida por Vasconcel $05^{(2)}$, é o que sustenta a elaboração de um projeto assistencial comum, como

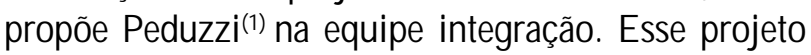
sinal iza a integração da equipe, na medida em que se constitui como um eixo em torno do qual se organiza a dinâmica cotidiana de trabalho.

Esse projeto, por sua vez, será sempre referido a uma determinada equipe e estará situado num determinado contexto sócio-histórico - micro e macro determinado. A ssim, o projeto geral de superação do modelo biomédico psiquiátrico pelo de atenção psicossocial no Brasil (processo atravessado pelos movimentos contrários à R eforma Psiquiátrica, localizados principalmente nas corporações médicas) e as específicas configurações locais das equipes dos CAPS estarão implicados e serão constituintes dos projetos assistenciais das equipes de cada CAPS. A historicidadeé, portanto, fundamental para compreender - trabalho em equipe na perspectiva da interdisciplinaridade (2 ou da equipe integração(1).

As equipes dos CAPS operam diferentes instrumentos de trabal ho - recursos materiais (desde a própria estrutura física dos serviços), conhecimentos especializados das diferentes áreas profissionais, técnicas (oficinas artísticas, culturais, educativas, de geração de renda; grupos terapêuticos; consultas médica, de enfermagem, psicologia,) e são, também elas próprias, simultaneamente meios/recursos e agentes do processo de trabalho. Nos CAPS, todos os agentes e meios necessitam ser coordenados para a finalidade de transformar o "objeto" de trabalho, os usuários dos CA PS, realizando a reabilitação psicossocial.

A necessidade da interdisciplinaridade no campo da Saúde M ental decorre do fato de que o "adoecer psíquico" não é um fenômeno homogêneo e simples, mas complexo e multifacetado. 0 sofrimento psíquico é decorrente de uma complexa teia de eventos biológicos, sociais, emocionais, psicológicos, culturais e políticos. Sendo assim, as formas de atenção a ele, para o seu arrefecimento, necessitam ser as mais diversificadas possíveis. 0 mero envolvimento de técnicos dediferentes formações, a justaposição de ações, freqüentemente antagônicas, característica da equipe multiprofissional, 
não resulta em melhoria da atenção, que só pode se beneficiar de ações integralizadas e contextual izadas ${ }^{(15)}$.

A partir da concepção de que cada manifestação de sofrimento psíquico ocorre de modo singular e que a finalidade da intervenção psicossocial não é mais o retorno a uma pretendida normalidade externa à pessoa, como ocorria no model o psiquiátrico, o campo da saúde mental é chamado a refazer-se por inteiro. $A$ historicidade dos processos individuais de adoecimento/reabilitação e de organização dos processos de trabal ho requer novas habilidades e competências das equipes.

A atenção psicossocial apresenta especificidades e um grau de complexidade de âmbito operacional, de gestão, de concepção teórica de objeto e de finalidade do trabal ho que necessitam ser considerados ao se buscar sua implantação. As equipes precisam de uma mínima coesão ideológica e ética(15).

Tendo a equipe tal importância para o trabalho dos CAPS, uma vez que a inovação aí proposta se dá (ou não) mediante a realização do "trabalho vivo em ato" ${ }^{\prime(5)}$, consideramos fundamental refletir sobre as condições a partir das quais este trabalho se realiza.

\section{PRECARIZAÇÃO DO TRABALHO E SUAS IM PLICAÇÕES NA ATENÇÃO PSICOSSOCIAL}

0 projeto neoliberal, conforme análise do contexto latino-americano ${ }^{(16)}$, reivindica um Estado mínimo, uma desobrigação cada vez mais acentuada das garantias trabal histas e de bem estar social, em um contexto onde essas garantias, a rigor, nunca existiram. Dentre as conseqüências desse projeto, observou-seno B rasil e em outros países, o enfraquecimento dos coletivos de trabalhadores e a acentuação da exploração, com a "subproletarização do trabal ho, presente nas formas de trabalho precário, parcial, temporário, subcontratado, tercerizado, vinculados à economia informal" (17:44).

Essa metamorfose e desagregação da classe trabalhadora atual(17) é evidenciada no setor saúde brasileiro de modo muito acentuado. A s conseqüências desse processo podem ser observadas em estudos que anal isam o processo de trabal ho de enfermagem ${ }^{(18)}$. N esse artigo, enfocamos um aspecto específico da precarização das condições de trabal ho - os contratos temporários -, na sua rel ação com a possibilidade (ou não) de construção de uma nova tecnologia na atenção à saúde mental - os CAPS.

A Portaria n. 336/GM/02 estabelece a equipe mínima de trabal ho para os CAPS detipo I, II, III,CAPSi e CAPSad, além de descrever as atividades que devem ser realizadas por essas equipes(11).

Tomando-se por base o CA PS de menor nível de complexidade previsto nesta mesma portaria, CAPS I, temos que:

\begin{abstract}
Para 0 atendimento de vinte pacientes por turno, tendo como limite máximo trinta pacientes por dia, em regime de atendimento intensivo, a equipe deverá estar composta por 1 médico com formação em saúde mental, 1 enfermeiro, 3 profissionais de nível superior entre as seguintes categorias: psicólogo, assistente social, terapeuta ocupacional, pedagogo ou outro profissional necessário ao projeto terapêutico; 4 profissionais de nível médio (técnico de enfermagem, administrativo ou educacional e artesão) ${ }^{(11: 32)}$.
\end{abstract}

Os demais CAPS têm uma conformação de equipe profissional semelhante, variando somente em número de profissionais e em algumas especialidades.

Por meio da abordagem dialética marxista estamos problematizando e analisando essas condições de trabal ho e suas implicações no trabalho das equipes dos CAPS.

$\mathrm{Na}$ perspectiva marxista a realidade é interpretada como uma totalidade onde tanto os fatores visíveis como as representações sociais integram e configuram um modo de vida condicionado pelo modo de produção específico(19:34).

A ssim, é destacada a base material como determinante da produção da consciência e construção da real idade, e a historicidade, pois a sociedade está sob o movimento do modo de produção econômico, que lhe dá direção num determinado momento e lugar ${ }^{(19)}$. A o considerarmos a atenção psicossocial, é necessário admitir que existe um intermediário - institucional, jurídico-político, legal - entre o trabalhador da saúde mental e o seu trabal ho. A tualmente, ao situar a existência dos CAPS no contexto do SUS, reconhece-se a preponderância de serviços de natureza pública. Esse intermediário, portanto, é o gestor público, na maioria das vezes situado em nível municipal.

\section{Reconhecendo que}

qual quer que seja o programa terapêutico que se crie [...], ele será profundamente sensível aos modos estruturais e conjunturais de organização do dispositivo institucional em que ou por meio do qual é executado (6:149)

é necessário considerar a precarização do trabalho em saúde como algo que terá influência central nos resultados do trabalho terapêutico nos dispositivos de atenção à saúde mental.

Para que o vínculo na equipe se instale e perdure, 
condições mínimas de trabalho devem ser asseguradas, como a estabilidade dos contratos de trabalho, bem como um salário condizente com cada categoria profissional, emanando daí, segurança do ponto de vista trabal hista. U ma equipe contratada precariamente (terceirizados, contratos temporários, por exemplo) não se responsabiliza pela missão institucional e muito menos se compromete com movimentos de mudança ${ }^{(20)}$, como é requisitado na atenção psicossocial.

Dentre as principais evidências sinalizadas pelos mercados de trabalho em saúde, em uma pesquisa realizada pelo M inistério da Saúde em 1995, foi a precarização dos mercados de trabal ho de profissionais da saúde ${ }^{(20)}$. Para exemplificar esta situação, encontramos que numa Secretaria M unicipal de Saúde de uma capital de Estado, o quadro de trabalhadores, no ano de 2004, se apresentava da seguinte forma: entre os 5.235 profissionais das mais diversas categorias, $46,3 \%$ eram estatutários (núcleo da carreira) e 50,1\% prestadores de serviços (contratos temporários) (21)

No documento preparatório para a 3a Conferência Nacional de Gestão no Trabalho e Educação na Saúde(2:36), realizada em 2005, encontramos que:

[...] a partir da década de 90, com a implementação da proposta neoliberal de reforma estrutural do Estado em direção ao Estado M ínimo, foi intensificado no SU S a política de flexibilização das contratações e de precarização das relações de trabalho, utilizando diversos model os de contratos de trabal ho adotados pela União, estados e municípios.

São importantes outros pontos deste mesmo documento: A terceirização de serviços passou a ser efetuada sem a observação da legislação, induzindo a propagação do trabalho precarizado; o trabalho precário está relacionado à desobediência de normas vigentes nos direitos administrativos trabal histas. Define-se trabalho precário como uma situação de não-proteção social, quando exercido na ausência dos direitos previstos na legislação ${ }^{(22)}$.

Observa-se ainda:

[...] como conseqüência da precarização do trabal ho, [que] um grande contingente de profissionais do setor público de saúde está submetido às mais diversas modalidades contratuais: cooperativas, sistemas de bolsas, contratos temporários, pagamento por reconhecimento de dívida, entre outros. Essas modalidades além de contrariarem os preceitos do artigo 60 da Constituição Federal, que garante 0 trabal ho como um direito social, submete esses profissionais a uma situação de desconforto e de insegurança trabalhista e social ${ }^{\text {(22: 37). }}$
A complexidade de composição e de objetivos que se apresentam para as equipes dos CAPS, requisitando a articulação dos diferentes processos de trabalho na constituição de um processo peculiar, certamente é, por si só, um grande desafio a ser enfrentado. M aior desafio ainda é articular os diferentes processos de trabal ho presentes nas equipes com as dificuldades impostas pela situação da precarização do trabal ho nos serviços públicos de saúde, especificamente nos serviços de saúde mental.

Outros estudos também apontaram a terceirização como uma dificuldade para o trabalho de enfermagem em outros setores, implicando em prejuízos para a qualidade da assistência(23).

\section{CONSIDERAÇÕES FINAIS}

Vivenciando os efeitos da precarização do trabalho, participando da construção da atenção psicossocial em nível municipal e amparadas nas referências teórico-metodológicas apontadas, consideramos que, se o que se pretende é a inversão do modelo asilar para o psicossocial, é necessário a coerência administrativa da gestão pública nesse sentido.

A pesar de reconhecermos outras formas de manifestação da precarização do trabalho tais como: número reduzido e baixo investimento em qualificação dos trabalhadores, condições internas desfavoráveis (ausência ou insuficiência de materiais e instrumentos), ausência de perspectivas de progressão no trabalho, ou seja, existência de carreiras, etc, nos limitamos, neste artigo, a refletir sobre a questão da estabilidade do trabal ho por ser de maior visibilidade no nível municipal que ora nos referimos, que teve seu último concurso público em 2001(24).

Consideramos que a força do projeto neoliberal se impõe no contexto anal isado. A ssim, apesar da ênfase normativa/legal de construção da Reforma Psiquiátrica e, consequentemente, reordenação da atenção em saúde mental no Brasil, o que se observa são os limites à realização dessa tecnologia, dados pelas políticas centrais de privilégio do capital.

Destacamos que providências radicais devam ser tomadas para a construção de uma cultura psicossocial: nos campos da formação, da admissão e da participação dos trabalhadores dos planos e projetos de gestão referentes à política de saúde mental. Mais explicitamente, nos referimos ao efetivo comprometimento dos gestores na formação de profissionais para a saúde e saúde mental; na realização sistemática de concursos públicos e com direcionamento 
dos conteúdos ao serviço específico que será prestado por cada categoria profissional. E, por fim, considerar a construção das ações de saúde como resultante de uma pactuação social que envolve usuário, trabalhadores e gestores.

\section{REFERÊNCIAS}

1. Peduzzi M . Equipe multiprofissional de saúde: conceito e tipologia. Rev Saúde Públ 2001; 35(1):103-9.

2. Vasconcelos EM . Serviço Social elnterdisciplina-ridade: o exemplo da saúde mental. In: VA SCONCELOS EM, organizador. Saúde M ental eServiço Social: 0 desafio da subjetividadee da interdiscipli-naridade. $2^{a}$ ed. São Paulo: Cortez; 2002. p. 35-65

3. Almeida M CP, Rocha SM . 0 trabalho de Enfermagem. São Paulo: Cortez; 1997.

4. M endes-Gonçalves RB. Práticas de saúde: processo de trabalho e necessidades. Cadernos Cefor. Prefeitura M unicipal de São Paulo. São Paulo: Secretaria M unicipal de Saúde; 1992.

5. Malta DC, M erhy EE. A micropolítica do trabalho em saúde: Revendo alguns conceitos. R ev M in Enferm 2003. 7(1):61-6.

6. Costa-R osa A. 0 modo Psicossocial: U m paradigma das práticas substitutivas ao modo asilar. In: A marante $P$, ornaizador. Ensaios: subjetividade, saúde mental, sociedade. Rio de J aneiro: FIOCRUZ, 2000.

7. Oliveira AGB, ALESSI NP. Superando o manicômio? Desafios na construção da reforma psiquiátrica. Cuiabá: EdUFM T; 2005.

8. A marante P. A (clínica) e a reforma psiquiátrica. In A marante $P$, coordenador. Arquivos de saúde mental e A tenção Psicossocial. Rio de Janeiro: NA U; 2003.

9. Organização Mundial da Saúde. Organização Panamericana deSaúde. Relatório sobrea saúde no mundo - saúde mental: nova concepção, nova esperança. Genebra; 2001.

10. Onocko-Campos RT, Furtado JP. Entre a saúdecoletiva e a saúde mental: um instrumental metodológico para avaliação da rede de Centros de A tenção Psicossocial (CA PS) do Sistema Único de Saúde. Cad Saúde Públ 2006; 22(5):1053-62.

11. Ministério da Saúde (BR). Saúde mental no SUS: os centros de atenção psicossocial. B rasília F; 2004.

12. A marante PDC, Torre EHG. A constituição de novas práticas no campo da atenção psicossocial: análise de dois projetos pioneiros na Reforma Psiquiátrica no Brasil.
Saúde em Debate 2001; 25:26-34.

13. OliveiraA GB. Trabal ho e cuidado no contexto da atenção psicossocial: al gumas reflexões. Rev Escola Enferm A nna Nery. No prelo 2006.

14. Peduzzi M, Palma JJL. A equipe de saúde. In: Schraiber LB, N emes M IB , organizador. Saúde do adulto: programas e ações na Unidade Básica. São Paulo: Hucitec; 2000.

15. Oliveira FB, SilvaA 0 . Enfermagem em Saúde M ental no contexto da reabilitação psicossocial e da interdisciplinaridade. Rev B ras Enferm 2000: 584-92.

16. Lurell $A C$, organizador. Avançando em direção ao passado: a política social do neoliberalismo. In: Estado e Políticas Sociais no Neoliberalismo. 2ª̣. São Paulo: Cortez; 1997.

17. Antunes R. A deus ao Trabal ho? Ensaio sobre as metamorfoses ea centralidade do mundo do trabal ho. São Paulo: Cortez; 1999.

18. Pires D, Gelbcke FL. Transformações no mundo do trabal ho e a enfermagem: transformações eoportunidades no mercado de trabal ho. In: A nais 53으을 de Enfermagem. Curitiba, 2001.

19. M inayo MCS. 0 desafio do conhecimento: pesquisa qualitativa em saúde. 3ạ ed. Rio de Janeiro: Hucitec/ A brasco; 1994.

20. Nogueira RP. Estabilidadeeflexibilização: tensão de base nas novas políticas de recursos humanos em saúde. Divulg Saúde Debate 1996; 14:18-22.

21. Secretaria M unicipal de Saúde de Cuiabá. Relatório de Gestão 2004. Cuiabá, 2005.

22. Ministério da Saúde (BR). Secretaria de Gestão do Trabalho e da Educação na Saúde. Documentos preparatórios para 3a C onferência $\mathrm{N}$ acional de Gestão do Trabalho e da Educação na Saúde. 2ª ed. B rasília. 2005.

23. Siqueira $\mathrm{HCH}$. A terceirização nos serviços e suas conseqüências no cuidar em enfermagem. CogitareEnferm 2005; 10(3).

24. Secretaria M unicipal de Saúde de Cuiabá. Relatório de Gestão 2005. Cuiabá, 2006. 